\title{
Development of Probability Matrices for Identification of Alaskan Marine Bacteria
}

\author{
A. W. DAVIS ${ }^{1}$ R. M. ATLAS,${ }^{1 *}$ AND M. I. KRICHEVSKY ${ }^{2}$ \\ Department of Biology, University of Louisville, Louisville, Kentucky 40292, ${ }^{1}$ and Microbial Systematics \\ Section, National Institute of Dental Research, Bethesda, Maryland $20205^{2}$
}

\begin{abstract}
Probabilistic identification matrices were developed by using data from 86 phenotypic clusters. In our analyses we used data gathered from 1,119 bacterial strains isolated from Alaskan outer continental shelf regions (320 features per isolate), the Jaccard similarity coefficient, and unweighted average linkage clustering. A normalized probability score (relative likelihood, identification score) based on a modification of the Bayes theorem and the ratio of observed likelihood to best possible relative likelihood were used as identification criteria. Error rates were compared for the proper association of isolates with 86 previously defined phenons by using an inclusive matrix of 61 feature probabilities and a hierarchical scheme containing a primary probability matrix (supermatrix) to indicate the most likely of six secondary probability matrices (submatrices) for detailed identification. The supermatrix-submatrix scheme was superior to the inclusive scheme on the basis of economy of tests and had a comparable error rate.
\end{abstract}

Alaskan outer continental shelf bacterial isolates were previously characterized by determining 320 features per strain $(7,11)$. The organisms characterized in these studies included eurytolerant, multisubstrate-utilizing species from sites in the Northeast Gulf of Alaska, psychrophilic, stenohaline, complex growth factor-requiring species from the Northwest Gulf of Alaska, and highly pleomorphic, psychrotrophic species from the Beaufort Sea. Few of the isolates from the Beaufort Sea and none of the isolates from the Gulf of Alaska could be closely associated with previously described marine bacteria, nor could these isolates be identified by existing probability matrices. The dominant organisms from the Gulf of Alaska and the Beaufort Sea were identified as members of the Moraxella-Acinetobacter, Beneckea-Vibrio, Microcyclus, and Flavobacterium groups, but were not identified to the species level. Several other Cytophaga, Pseudomonas, Alcaligenes, Alteromonas, and coryneform species also were found. Most isolates could be placed into one of the following seven categories: (i) gram-negative rods producing yellow or orange pigments (Flavobacterium, (ytophaga); (ii) gram-negative, full or partial ring-forming organisms (Microcyclus); (iii) gram-negative, nonpigmented, fermentative, facultative anaerobic, curved or straight rods (Vibrio); (iv) gram-negative, nonpigmented, weakly fermentative, facultatively anaerobic, pleomorphic rods (unidentifiable with any genus described in Bergey's Manual of Determinative Bacteriology, 8th ed. [2]); (v) gram-negative, nonpigmented, nonfermentative, aerobic rods (Pseudomonas, Alcaligenes, Alteromonas); (vi) non-acid-fast coryneform bacteria showing branching and snapping division (Arthrobacter); and (vii) gram-negative coccobacilli (Acinetobacter).

The object of this study was to construct an original set of identification matrices based on the minimal number of easily and objectively performed tests for phenetic characteristics. Computer-assisted numerical methods for identification of bacteria have been described previously by Willcox et al. $(16,17)$, Bascomb et al. (1), Dybowski and Franklin (3), Friedman and MacLowry (5), Friedman et al. (4), Gyllenberg (6), and Lapage et al. $(12,13)$.

\section{MATERIALS AND METHODS}

Strain characterization and selection. Based on numerical taxonomic analyses of nine previously described data sets, which included data on 4,200 strains $(7,11)$, groups of four or more strains with minimal similarity levels of $70 \%$, as determined by the Jaccard similarity coefficient and single linkage clustering, were selected for this study. A total of 1,206 strains (representing 108 clusters) were selected. All nine data sets were combined, and the data were edited to exclude features for which all of the strains gave results greater than or equal to $90 \%$ positive, $90 \%$ negative, or $50 \%$ unknown, thereby eliminating tests with low discriminating power. A series of cluster analyses was performed by using the Jaccard coefficient and unweighted average linkage to assess the integrity of the original clusters. Five overlapping analyses (600 strains each) were performed to include 
the entire data set. The criterion for inclusion of strain clusters was relaxed to $60 \%$ similarity because of the substitution of average linkage for single linkage clustering. Clusters observed to have lost members, so that the number of strains in the new cluster was less than four, were eliminated from the study; original clusters incorporating new members were redefined to include these new strains.

Taxonomic analyses. Of the 1,206 strains originally selected from the clusters defined in the original studies, 1,119 clustered in the same manner when the data from multiple sets were combined and unweighted average linkage was used. Strains lost from individual clusters (total, 87 strains) either appeared as outliers linked to members of the same original clusters at levels of less than $60 \%$ overall similarity or clustered above the $60 \%$ criterion in groups of three strains or less. We observed no instances in which an original cluster established by using the Jaccard similarity coefficient and the single linkage clustering algorithm gained strains from other clusters initially defined within the same primary study. Based on the results of unweighted average linkage, 1,119 strains in 86 clusters were chosen for our final taxonomic analyses.

Character editing and selection. Of the original 320 previously described tests $(7,11)$, those which were highly reproducible (i.e., which were easy to prepare, to carry out, and to read), those which were most objective, and those which tested for as many different enzymes and biochemical pathways as possible were retained for matrix construction. Characteristics subject to excessive investigator bias and tests requiring extensive time to perform (i.e., tests that are unreasonable to include for routine identification) were eliminated from the set of eligible group descriptors for development of probabilistic identification matrices. Additionally, specific descriptors of colony pigmentation were reduced to a single test describing whether colonies were pigmented or nonpigmented. In this manner 92 tests were eliminated, leaving 228 features in the data set; these were primarily metabolic and physiological features for potential use as phenon discriminators.

Construction of identification matrices. The discriminatory power of the 228 features was assessed by calculating the group partitioning index (GPI), using the CORR program (14a). This index is similar in intent to the separation index of Gyllenberg (6) but simpler to calculate. Since previous reports $(7,10,11)$ supported the use of salt requirement, growth at $25^{\circ} \mathrm{C}$,

TABLE 1. Group feature frequencies for three attributes

\begin{tabular}{cccc}
\hline Group & \multicolumn{3}{c}{ \%o of strains } \\
\cline { 2 - 4 } & $\begin{array}{c}\text { Absence of } \\
\text { pigmentation }\end{array}$ & $\begin{array}{c}\text { Growth at } \\
25^{\circ} \mathrm{C}\end{array}$ & $\begin{array}{c}\mathrm{NaCl} \\
\text { requirement }\end{array}$ \\
\hline 1 & 92 & 94 & 89 \\
2 & 95 & 98 & 36 \\
3 & 96 & 33 & 99 \\
4 & 95 & 1 & 1 \\
5 & 5 & 87 & 89 \\
6 & 1 & 81 & 25 \\
7 & 2 & 10 & 72 \\
8 & 2 & 11 & 40 \\
\hline
\end{tabular}

and lack of pigment production (i.e., gray colonies) in determinative schemes, these features were chosen from among those having the highest GPIs. All 86 clusters were examined for the results of these tests. Note that lack of pigmentation was used because the original features that were scored contained tests for 17 different pigments; lack of pigment production indicates nonproduction of any of the 17 pigments. The use of absence of pigment as a positively scored test results from the original, common practice of scoring a battery of possible colony colors (including gray) as mutually exclusive. Thus, each feature is the complement of all other pigment feature frequencies combined. To avoid errors and simplify the computer manipulations of the data, we chose to retain nonpigmented colonies as a positive attribute; mathematically, the choice is arbitrary.

Three attributes allow eight possible combinations of results (Table 1). However, because of the variability of groups 1 and 2 and groups 7 and 8 for salt requirements, these group pairs were combined, and a new feature frequency output was generated for distinguishing six groups; the resultant matrix was supplemented with two additional tests, growth in a medium supplemented with $0.5 \% \mathrm{NaCl}$ and Tween 20 hydrolysis, which permitted further separation of additional subset pairs (Table 2). The supermatrix was designed to assign strains to proper submatrices for identification as members of defined phenons. Any phenon having variable results for one or more tests was placed in the group that most closely approximated its behavior for the variable test. For example, a taxon for which the percentages of strains positive in the tests for lack of pigmentation, growth at $25^{\circ} \mathrm{C}$, and salt requirement were 98,88 , and $14 \%$, respectively, would be placed in group 2, whereas a taxon for which the percentages of strains positive in the same tests were 96,88 , and $53 \%$, respectively, would be placed in group 1 .

To accomplish the probabilistic identification of strains belonging to the original 86 clusters, it was necessary to develop six separate submatrices. Additionally, an inclusive matrix containing all 86 clusters was developed for comparison. The contents of each submatrix were determined by the set of clusters contained in the respective group of the supermatrix and the adequate number of features required to completely separate each cluster pair within the same group. Features were selected based on their discriminatory power by using the CORR program (14a) to

TABLE 2. Feature frequencies for modified groups with five features

\begin{tabular}{cccccc}
\hline Group(s) & $\begin{array}{c}\text { Absence } \\
\text { of } \\
\text { pigmen- } \\
\text { tation }\end{array}$ & $\begin{array}{c}\text { Growth } \\
\text { at } 25^{\circ} \mathrm{C}\end{array}$ & $\begin{array}{c}\text { NaCl re- } \\
\text { quire- } \\
\text { ment }\end{array}$ & $\begin{array}{c}\text { Tween } \\
20 \text { hy- } \\
\text { drol- } \\
\text { ysis }\end{array}$ & $\begin{array}{c}\text { Growth } \\
\text { in } 0.5 \% \\
\mathrm{NaCl}\end{array}$ \\
\hline 1,2 & 97 & 95 & 73 & 75 & 90 \\
3 & 95 & 1 & 99 & 92 & 1 \\
4 & 95 & 1 & 1 & 72 & 15 \\
5 & 6 & 95 & 95 & 39 & 90 \\
6 & 1 & 99 & 4 & 27 & 92 \\
7,8 & 1 & 8 & 60 & 8 & 80 \\
\hline
\end{tabular}


determine the minimal number of features required to completely partition each possible cluster pair within its respective subset. The CORR program ranks tests in order of decreasing partitioning value. Correlation and redundancy measures of all appropriate features were calculated for the cluster combinations within each submatrix to minimize duplication. Features were chosen individually by decreasing rank of GPI such that each cluster pair combination was separated by at least two tests that differed by a minimum of $60 \%$ in positive feature frequency. In cases where two tests had the same separation value, the test with the least overall redundancy with previously chosen features was selected. The numbers of clusters and features contained in each matrix are shown in Table 3 . The design criterion that at least two tests completely separate all possible cluster pairs within the given subset was met by using a total of 52 tests in the supermatrix-submatrix scheme (Table 4). The inclusive matrix required 61 features to separate the 86 clusters. Thus, nine features required for the complete separation of all group pairs in the inclusive matrix were not required in any of the supermatrix-submatrix combinations; these included cell length of 0.5 to 1.0 $\mu \mathrm{m}$, ammonia production, cellobiose utilization with production of acid, glycerol utilization, acetic acid utilization, alpha-ketoglutaric acid utilization, and Lmethionine utilization.

Evaluation technique. The supermatrix, the six submatrices, and the inclusive matrix were incorporated into the IDDNEW identification program (8). To evaluate the probability matrices developed in this study, data for the 1,119 Alaskan marine strains were used. The threshold identification level set by the IDDNEW program is 0.999 ; i.e., for a strain to be identified as a member of a taxon, its identification (ID) score must be 1,000 times greater than the ID score of the next most probable taxon. This is the level used by Lapage et al. (13), who presented data on the way in which varying the ID score threshold levels affected the percentage of strains identified to the correct taxon and the percentage identified to the incorrect taxon. These authors found a decrease of approximately 2 to $3 \%$ and an increase of about $0.1 \%$ in the two rates, respectively, when the ID score threshold for identification was lowered from 0.999 to 0.970 . When both the ratio of observed absolute likelihood to best possible absolute likelihood ( $\mathrm{R}$ score) and the ID score are used for identification, the rate of incorrect identification is diminished; this is what we did. In our study, for assignment to a submatrix the threshold for identification was ignored. The most probable submatrix was chosen for each test strain regardless of the probabilities for less likely submatrices. The numbers of correct and incorrect submatrix assignments were calculated. Strains having insufficient data (additional tests were suggested when the strains were analyzed by using the supermatrix) were not submitted to submatrices as no error rate could be calculated for these strains. The results from the submatrices were evaluated to determine the percentage of strains that were properly assigned to a given submatrix which identified with its group of origin. All correct strain group identifications for which no additional tests were suggested were classified in terms of the actual ID scores; i.e., within each matrix, the ID scores of correct strain group identifications were tabulated. In addition to ID scores for evaluation of matrix efficiency, $\mathrm{R}$ scores were calculated. $R$ scores have been described by Wayne et al. (15) for the evaluation of probability matrices developed for identification of mycobacteria.

The efficiencies of a matrix for identification were calculated from the distribution of the strains that exhibited ID and $\mathrm{R}$ scores above the following thresholds: ID score of 0.990 and $R$ score of 0.01 ; and ID score of 0.970 and $R$ score of 0.001 . The supermatrix efficiency was evaluated by determining the percentage of correct submatrix assignments of test strains, regardless of ID scores. Submatrix efficiencies were evaluated by determining the percentage of strains identified in the correct group, regardless of ID scores, and the percentage of correct strain group identifications below or above either or both sets of criteria (i.e., ID score of 0.90 and R score of 0.01 ; and ID score of 0.970 and $R$ score of 0.001 ). Strains having the wrong group identification were examined for identification at these thresholds as well. Positive identification of any strain required that both the ID score and the $\mathrm{R}$ score were equal to or greater than the given criteria.

Error rates were calculated for each supermatrixsubmatrix combination ( $\mathrm{six}$ in all), and an overall error rate for all matrices was calculated as follows: $[(X+Y$ $+Z) / Q+R] \times 100=$ error rate, where $X$ is the total number of strains assigned to the correct group of origin for which ID and R scores were below threshold levels, $Y$ is the total number of strains which were assigned to the correct submatrix but which were identified above identification threshold levels as being members of the wrong group of origin, $Z$ is the total number of strains assigned to the wrong submatrix identified above identification thresholds, $Q$ is the valid number of strains submitted to the correct submatrix, and $R$ is the valid number of strains submitted to the incorrect submatrix. The matrices described here are available from us.

\section{RESULTS}

Of the 1,087 strains submitted to the supermatrix for assignment to appropriate submatrices, 17 were excluded from further testing as additional tests were suggested for these strains. Additionally, 36 of the test strains submitted to the battery of submatrices were found to have insufficient recorded test data for identification in the appropriate submatrices. Most of these strains $(86 \%)$ were assigned to submatrices 2 and 6 . The distribution of the 1,034 remaining test

TABLE 3. Probability matrix specifications

\begin{tabular}{clcc}
\hline Matrix & \multicolumn{1}{c}{ Name } & $\begin{array}{c}\text { No. of } \\
\text { features }\end{array}$ & $\begin{array}{c}\text { No. of } \\
\text { groups }\end{array}$ \\
\hline 1 & Supermatrix & 5 & 6 \\
2 & Submatrix 1 & 30 & 35 \\
3 & Submatrix 2 & 13 & 10 \\
4 & Submatrix 3 & 13 & 10 \\
5 & Submatrix 4 & 18 & 18 \\
6 & Submatrix 5 & 13 & 10 \\
7 & Submatrix 6 & 20 & 16 \\
8 & Inclusive matrix & 61 & 86 \\
\hline
\end{tabular}


TABLE 4. Features used in the various matrices

\begin{tabular}{|c|c|c|c|c|c|c|c|c|}
\hline \multirow{2}{*}{ Feature } & \multirow{2}{*}{$\begin{array}{l}\text { Inclusive } \\
\text { matrix }\end{array}$} & \multirow{2}{*}{$\begin{array}{l}\text { Super- } \\
\text { matrix }\end{array}$} & \multicolumn{6}{|c|}{ Submatrices } \\
\hline & & & 1 & 2 & 3 & 4 & 5 & 6 \\
\hline Cell length of 0.5 to $1.0 \mu \mathrm{m}$ & $x^{a}$ & & & & & & & \\
\hline Capsule produced & $\times$ & & & & & $x$ & & $\times$ \\
\hline Cell motile & $\times$ & & $x$ & & & & & \\
\hline Nondiffusible yellow pigment & $\times$ & & & & & $x$ & $x$ & $x$ \\
\hline Nonpigmented & $x$ & $\times$ & & & & & & \\
\hline Colonies $<1 \mathrm{~mm}$ in diameter after 10 days & $\times$ & & & & & & & $\times$ \\
\hline Colonies 1 to $2 \mathrm{~mm}$ in diameter after 10 days & $\times$ & & & & & & & $\times$ \\
\hline Colonies opaque & $x$ & & $x$ & & & & $\times$ & \\
\hline D-Glucose catabolized aerobically & $\times$ & & $x$ & & $\times$ & & & \\
\hline D-Glucose catabolized anaerobically & $x$ & & $x$ & $x$ & $x$ & & $\times$ & $\times$ \\
\hline Ammonia produced & $\times$ & & & & $x$ & & & \\
\hline Nitrate reduced & $\times$ & & & & & & & $x$ \\
\hline Oxidase produced (Kovacs) & $\times$ & & $x$ & $\times$ & & & & \\
\hline Acid from D-ribose & $\times$ & & & & $\times$ & $x$ & & \\
\hline Acid from $D$-fructose & $\times$ & & & $\times$ & $\times$ & $x$ & & \\
\hline Acid from cellobiose & $\times$ & & & & & & & \\
\hline Starch hydrolyzed & $\times$ & & $x$ & & & & $\times$ & $x$ \\
\hline Tween 20 hydrolyzed & $x$ & $\times$ & & & & & & \\
\hline Tween 80 hydrolyzed & $x$ & & $x$ & $x$ & $\times$ & & & \\
\hline Growth at pH 6.0 & $x$ & & & $x$ & & $\times$ & $\times$ & $\times$ \\
\hline Growth at $\mathrm{pH} 10.0$ & $\times$ & & $x$ & & & & & $x$ \\
\hline Growth at $20^{\circ} \mathrm{C}$ & $x$ & & & $x$ & & & & $x$ \\
\hline Growth at $25^{\circ} \mathrm{C}$ & $x$ & $\times$ & & & & & & \\
\hline $\mathrm{NaCl}$ required for growth & $x$ & $x$ & & & & & & \\
\hline Growth with $0.5 \% \mathrm{NaCl}$ & $\times$ & $\times$ & & & & & & \\
\hline Growth with $5.0 \% \mathrm{NaCl}$ & $\times$ & & & & & $\times$ & & $x$ \\
\hline Growth with $7.5 \% \mathrm{NaCl}$ & $\times$ & & $x$ & & & $x$ & $x$ & $x$ \\
\hline Growth with $10.0 \% \mathrm{NaCl}$ & $\times$ & & $x$ & & & $x$ & & \\
\hline D-Fructose utilized & $x$ & & & & & & & $x$ \\
\hline D-Galactose utilized & $x$ & & & & & & & $x$ \\
\hline D-Glucose utilized & $x$ & & $x$ & $x$ & & $x$ & & $x$ \\
\hline L-Sorbose utilized & $x$ & & $\times$ & & & $x$ & & \\
\hline Maltose utilized & $x$ & & $x$ & $x$ & & & $x$ & \\
\hline Glycerol utilized & $x$ & & & & & & & \\
\hline Acetate utilized & $x$ & & & & & & & \\
\hline Caprylate utilized & $x$ & & $x$ & & & & & \\
\hline Valerate utilized & $x$ & & $x$ & & & & & \\
\hline Glutarate utilized & $x$ & & $x$ & $x$ & & & & \\
\hline Fumarate utilized & $\times$ & & $x$ & & $x$ & & $x$ & \\
\hline Beta-hydroxybutyrate utilized & $\times$ & & $x$ & & & & & \\
\hline DL-Lactate utilized & $x$ & & $x$ & & & & & \\
\hline 2-Ketogluconate utilized & $x$ & & $x$ & & & & & \\
\hline Pyruvate utilized & $x$ & & & $x$ & & $x$ & & \\
\hline Alginate utilized & $\times$ & & & & & & & \\
\hline Benzoate utilized & $\times$ & & & & & & & \\
\hline$m$-Hydroxybenzoate utilized & $x$ & & $x$ & & & & & \\
\hline L-Aspartate utilized & $x$ & & & & & & & \\
\hline L-Methionine utilized & $x$ & & & & & & & \\
\hline L-Proline utilized & $x$ & & $\times$ & $x$ & $x$ & $\times$ & & \\
\hline L-Threonine utilized & $\times$ & & $x$ & & & & & \\
\hline Tryptone utilized & $\times$ & & $\times$ & $x$ & $\times$ & $\times$ & $x$ & $x$ \\
\hline Peptones utilized & $x$ & & & $x$ & $x$ & & $x$ & $x$ \\
\hline L-Glutamate utilized as sole $C$ source & $x$ & & & & $x$ & $\times$ & $x$ & $x$ \\
\hline Acetate utilized as sole $\mathrm{C}$ source & $\times$ & & $\times$ & & & $x$ & & \\
\hline Pyruvate utilized as sole $\mathrm{C}$ source & $\times$ & & $x$ & & $x$ & & & \\
\hline Succinate utilized as sole $\mathrm{C}$ source & $\times$ & & $x$ & & & & & \\
\hline Lactate utilized with vitamins & $x$ & & & & & $\times$ & & \\
\hline Ribose utilized with vitamins & $x$ & & $\times$ & & $\times$ & $x$ & & \\
\hline Pyruvate utilized with vitamins & $x$ & & $\times$ & & & & $x$ & \\
\hline Aspartate utilized with vitamins & $x$ & & & & & $x$ & $x$ & \\
\hline Undefined growth factors required & $x$ & & & & & & & $x$ \\
\hline
\end{tabular}

$a \times$, Feature included in matrix. 
TABLE 5. Distribution of test strains according to test submatrix and highest ID score

\begin{tabular}{|c|c|c|c|c|c|c|c|c|c|c|c|c|}
\hline \multirow[b]{2}{*}{ Matrix } & \multirow{2}{*}{$\begin{array}{l}\text { Total } \\
\text { no. of } \\
\text { strains } \\
\text { in }\end{array}$} & \multirow{2}{*}{$\begin{array}{c}\text { Total } \\
\text { no. of } \\
\text { strains } \\
\text { out }\end{array}$} & \multirow{2}{*}{$\begin{array}{c}\text { No. } \\
\text { of } \\
\text { tests } \\
\text { sug- } \\
\text { gested }\end{array}$} & \multicolumn{9}{|c|}{ Distribution of ID scores (no. of strains) } \\
\hline & & & & $\geq 0.999$ & $\begin{array}{c}<0.999 \\
\quad \text { but } \\
\geq 0.995\end{array}$ & $\begin{array}{c}<0.995 \\
\quad \text { but } \\
\geq 0.990\end{array}$ & $\begin{array}{c}<0.990 \\
\text { but } \\
\geq 0.980\end{array}$ & $\begin{array}{c}<0.980 \\
\quad \text { but } \\
\geq 0.970\end{array}$ & $\begin{array}{c}<0.970 \\
\quad \text { but } \\
\geq 0.960\end{array}$ & $\begin{array}{c}<0.960 \\
\quad \text { but } \\
\geq 0.950\end{array}$ & $\begin{array}{l}<0.950 \\
\quad \text { but } \\
\geq 0.900\end{array}$ & $<0.900$ \\
\hline \multicolumn{13}{|c|}{ Submatrices } \\
\hline 1 & 524 & 523 & 1 & $429(2)^{a}$ & 38 & 14 & 7 & 5 & $5(2)$ & $2(1)$ & 8 & $15(1)$ \\
\hline 2 & 58 & 51 & 7 & 35 & 6 & 0 & 1 & 4 & 0 & 1 & 1 & $3(1)$ \\
\hline 3 & 73 & 72 & 1 & 61 & 4 & 1 & 1 & 1 & 0 & 0 & 1 & $3(2)$ \\
\hline 4 & 133 & 131 & 2 & 100 & 14 & 7 & 2 & 1 & 0 & 0 & 0 & $7(1)$ \\
\hline 5 & 61 & 60 & 1 & 41 & 7 & $4(1)$ & 1 & 1 & 1 & 0 & 1 & $4(1)$ \\
\hline 6 & 221 & 197 & 24 & 103 & 39 & $15(1)$ & $19(1)$ & $5(1)$ & 1 & 2 & 5 & 8 \\
\hline $\begin{array}{c}\text { Inclusive } \\
\text { matrix }\end{array}$ & 1,087 & 1,073 & 14 & $1,015(2)$ & 26 & 7 & 5 & $5(1)$ & 3 & $2(1)$ & 2 & $8(4)$ \\
\hline
\end{tabular}

${ }^{a}$ The numbers in parentheses indicate the absolute proportions of cell frequency for which the highest relative likelihood indicated an incorrect strain group association.

strains, which were assigned to groups based on the highest ID score, is shown in Table 5. When the supermatrix-submatrix scheme was used 949 strains $(91.7 \%)$ had ID scores equal to or greater than 0.970 . When the inclusive matrix was used, 1,058 strains $(98.6 \%)$ had ID scores equal to or greater than 0.970 . Eight strains $(0.7 \%)$ entered in the inclusive scheme had ID scores below 0.900 , and only one-half of these strains were assigned to incorrect groups. In the supermatrixsubmatrix scheme, the ID scores for 40 strains (3.9\%) were less than 0.900 , but only 6 strains were assigned to incorrect groups.

When $\mathbf{R}$ scores were used to evaluate the efficiency of identification, $27.6 \%$ of the strains tested in the supermatrix-submatrix scheme had $\mathrm{R}$ scores of 1.0 , compared with $7.4 \%$ of the strains in the inclusive scheme (Table 6). The use of ID and R score thresholds of 0.990 and 0.01 , respectively, did not produce a significantly higher rate of misidentification than a threshold ID score of 0.999 , as dictated by the IDDNEW program (Table 7). When ID and R score thresholds of 0.990 and 0.01 , respectively, were used, only one additional strain was identified incorrectly. The super- and submatrices permitted identification of $92 \%$ of the strains; the inclusive matrix resulted in identification of $93 \%$ of the strains. Lowering the identification criteria to ID and $R$ scores of 0.970 and 0.001 , respectively, increased the identification rate in the supermatrix-submatrix scheme by $8 \%$, with misidentification of only one additional strain. Likewise, lowering the criteria for the inclusive matrix in the same manner increased the identification rate by $15 \%$, with misidentification of only two additional strains. The most significant difference in the identification rates achieved by the two sets of identification criteria was observed for strains associated with submatrix 5 . When these strains were run in the inclusive matrix, a $35 \%$ increase in identification was observed if the lower criteria (ID score of 0.970 and $\mathrm{R}$ score of 0.001 ) were used compared with the higher criteria (ID score of 0.990 and R score of 0.01 ).

Values were calculated for total numbers of strains correctly assigned to a given submatrix, strains resulting in a correct group identification, strains with correct group identifications which exceeded the threshold criteria, and strains resulting in incorrect group identifications which exceeded threshold criteria when the different matrices were used (Table 8). The values for percentages of error represent the combined probabilities of making an incorrect conclusion about the group association of an unknown strain and its fit within a group for each supermatrix-submatrix scheme and the inclusive scheme. Also included is a total error rate for the supermatrix-submatrix scheme. The lowest er-

TABLE 6. Distribution of test strains according to submatrix trial and $\mathrm{R}$ scores

\begin{tabular}{cccccc}
\hline & \multicolumn{5}{c}{ Distribution of R scores (no. of strains) } \\
\cline { 2 - 6 } Matrix & $10^{0}$ & $\begin{array}{c}<10^{0} \\
\text { but } \\
\geq 10^{-1}\end{array}$ & $\begin{array}{c}<10^{-1} \\
\text { but } \\
\geq 10^{-2}\end{array}$ & $\begin{array}{c}<10^{-2} \\
\text { but } \\
\geq 10^{-3}\end{array}$ & $\begin{array}{c}<10^{-3} \\
\text { but } \\
\geq 10^{-4}\end{array}$ \\
\hline Submatrices & 100 & $227(4)^{a}$ & $129(1)$ & $49(1)$ & 18 \\
1 & $37(1)$ & 12 & 2 & 0 & 0 \\
2 & 36 & 23 & 12 & 1 & 0 \\
3 & 61 & $55(1)$ & 11 & 3 & 1 \\
4 & 19 & $35(1)$ & $6(1)$ & 0 & 0 \\
5 & $32(1)$ & $89(1)$ & 60 & 11 & $5(1)$ \\
6 & 79 & $470(3)$ & $286(3)$ & $170(2)$ & 68 \\
Inclusive & & & & & \\
matrix & & & & & \\
\hline
\end{tabular}

${ }^{a}$ Numbers in parentheses indicate the absolute proportions of cell frequency for which the highest relative likelihood indicated an incorrect strain group association. 


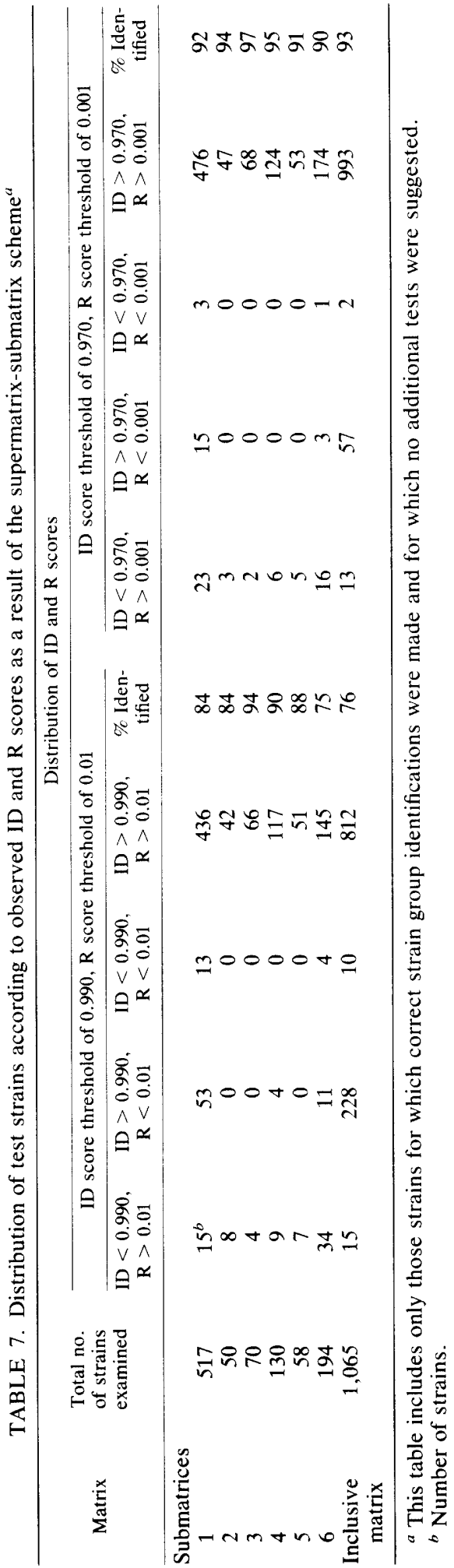

ror rate was observed for submatrix 3 when the lower set of criteria was used; the highest error rate was observed for submatrix 6 when the less lenient identification thresholds were used. The greatest difference in error rate across both identification threshold sets was observed for the inclusive matrix; i.e., the inclusive matrix was $16 \%$ more efficient when the lower rather than the higher identification threshold criteria were used. The error rate for the overall submatrix scheme was $7.6 \%$ less than that observed for the inclusive matrix when the higher threshold criteria were used; however, the submatrix scheme was slightly less efficient than the inclusive scheme when the lower threshold criteria were used ( 8.2 versus $6.9 \%)$.

\section{DISCUSSION}

Studying the numerical taxonomy of bacteria from the Gulf of Alaska, Hauxhurst et al. (7) had difficulty clearly separating genera based on the use of arithmetically defined key features. Several attempts to separate groups based on several different combinations of features with the highest GPIs failed to yield clear separation of the groups until we tried growth at different concentrations of $\mathrm{NaCl}$, at different temperatures, and at different $\mathrm{pH}$ values and the distribution of pigmented bacterial strains were cited as important discriminators for the differentiation of Gulf of Alaska isolates by Hauxhurst et al. (7). Similarly, the work of Kaneko et al. (10, 11) supported the use of the parameters given above for grouping marine bacteria from the Beaufort Sea. The requirement for $\mathrm{NaCl}$ separates marine bacteria from nonmarine bacteria, and growth at $25^{\circ} \mathrm{C}$ separates psychrophilicpsychrotrophic populations from mesophilic populations. These features had GPIs just below the highest rank. Thus, they were chosen for further investigation on a combination of historical (subjective) and numerical grounds. These features were used to divide the spectrum of taxonomically defined groups into subsets of workable sizes. Subsequent choices of highly discriminating characters within subgroups were based entirely on the arithmetically determined measures of separation values, character correlation, and redundancy.

Since the success of identification procedures depends on minimizing errors, we should consider the sources of potential error in the procedures used in this study. Errors may result from inaccurate data acquisition, poor sampling of the phenetic hyperspace, shortcomings of the clustering algorithm, and inappropriate threshold levels for probabilistic identification (14). Hauxhurst et al. (7) found a testing error rate of $3 \%$ for Gulf of Alaska isolates based on repetitive test- 
TABLE 8. Error rates for various matrices

\begin{tabular}{|c|c|c|c|c|c|c|c|c|c|c|}
\hline \multirow[b]{2}{*}{ Matrix } & \multicolumn{2}{|c|}{$X^{a}$} & \multicolumn{2}{|c|}{$Y^{b}$} & \multicolumn{2}{|c|}{$Z^{c}$} & \multirow[b]{2}{*}{$Q^{d}$} & \multirow[b]{2}{*}{$R^{e}$} & \multicolumn{2}{|c|}{ Error rate $(\%)$} \\
\hline & $\begin{array}{l}\text { Thresh- } \\
\text { old } 1^{f}\end{array}$ & $\begin{array}{l}\text { Thresh- } \\
\text { old } 2\end{array}$ & $\begin{array}{l}\text { Thresh- } \\
\text { old } 1\end{array}$ & $\begin{array}{l}\text { Thresh- } \\
\text { old } 2\end{array}$ & $\begin{array}{l}\text { Thresh- } \\
\text { old } 1\end{array}$ & $\begin{array}{l}\text { Thresh- } \\
\text { old } 2\end{array}$ & & & $\begin{array}{l}\text { Thresh- } \\
\text { old } 1\end{array}$ & $\begin{array}{l}\text { Thresh- } \\
\text { old } 2\end{array}$ \\
\hline \multicolumn{11}{|c|}{ Submatrices } \\
\hline 1 & 81 & 41 & 2 & 2 & 0 & 0 & 523 & 1 & 15.8 & 8.2 \\
\hline 2 & 8 & 2 & 0 & 0 & 0 & 0 & 51 & 0 & 15.7 & 3.9 \\
\hline 3 & 4 & 2 & 0 & 0 & 0 & 0 & 72 & 2 & 5.4 & 2.7 \\
\hline 4 & 13 & 6 & 0 & 0 & 1 & 2 & 131 & 10 & 9.9 & 5.7 \\
\hline 5 & 7 & 5 & 1 & 1 & 4 & 5 & 60 & 16 & 15.8 & 14.5 \\
\hline 6 & 49 & 20 & 0 & 1 & 0 & 0 & 197 & 3 & 24.5 & 10.5 \\
\hline $\begin{array}{c}\text { Inclusive } \\
\text { matrix }\end{array}$ & 253 & 72 & 1 & 3 & & & 1,073 & & 23.7 & 6.9 \\
\hline
\end{tabular}

${ }^{a} X$, Total number of strains assigned to the correct group of origin for which ID and $\mathrm{R}$ scores were below threshold levels.

${ }^{b} Y$, Total number of strains which were assigned to the correct submatrix but which were identified as members of the wrong group of origin above identification threshold levels.

${ }^{c} Z$, Total number of strains assigned to the wrong submatrix identified above identification thresholds.

${ }^{d} Q$, Valid number of strains submitted to the correct submatrix.

${ }^{e} R$, Valid number of strains submitted to the incorrect submatrix.

${ }^{f}$ Criteria for threshold $1:$ ID score $\geq 0.990$ and $R$ score $\geq 0.01$. Criteria for threshold 2 : ID score $\geq 0.970$ and $R$ score $\geq 0.001$.

ing of representative strains in duplicate tests. The total error rates for the supermatrix-submatrix and inclusive schemes described in this study were 8 and $7 \%$, respectively, for lenient identification thresholds (ID score greater than or equal to 0.970 and $\mathrm{R}$ score equal to 0.001 ). If the inherent testing error rate suggested by Hauxhurst et al. (7) holds for the testing of Alaskan outer continental shelf marine isolates in general, the proportions of unexplained error are only 5 and $4 \%$ for the supermatrix-submatrix procedure and the inclusive matrix procedure, respectively; the residual $3 \%$ error was presumably due to errors in the test procedures used to acquire the data.

To account for this residual error, we combined the occurrence of outlier and transition strains. Such peripheral strains should exhibit low likelihood scores even for their original phenons, especially since the character set on which similarity was originally estimated had been significantly reduced. Of the four strains assigned to wrong groups and falling above the identification threshold criteria after the supermatrix, two strains were peripheral strains of large phenons; similarly, two of the three strains misidentified by the inclusive scheme were peripheral members of large phenons. The remaining misidentified strains were inadequately described because of large amounts of missing data.

We also note that the choice of the particular ID and R score thresholds used as conclusive identification criteria depends on the purpose for which the identification matrices are employed. Wayne et al. (15) used an ID score threshold of 0.990 and an $\mathrm{R}$ score threshold of 0.01 for medically important mycobacteria. However, the use of lower threshold criteria in this study permitted a significantly higher identification rate for outer continental shelf isolates, with a concurrent increase in the misidentification rate of only two additional strains compared with the misidentification rate observed under the stringent criteria. Under strict identification criteria, identification and misidentification rates are lowered. This may be desirable in medical diagnostic laboratories as erroneous conclusions about the nature of a particular microorganism may have serious consequences. In instances where human health is not a consideration, however, lowering the identification threshold levels increases the efficiency of identification, with perhaps only a slight increase in identification error rate. Higher rates of natural variability in nonstressed ecosystems where the selective pressure does not force the emergence of highly genetically restricted populations require the use of lower threshold levels for identification. In many nonclinical situations the use of lowered identification thresholds that result in increased identification rates is advantageous.

Before this study, there was no documented use of super- and submatrix arrangements for probabilistic identification. Our results support the conclusion that this type of arrangement permits a considerable increase in experimental economy. In this study, the supermatrix-submatrix scheme for identification purposes used a maximum of $16 \%$ of the original set of binary information to identify a strain. The supermatrix-submatrix scheme may require that as few as 18 tests be performed on a set of unknown organisms for conclusive identification, com- 
pared with the 61 tests required by the inclusive matrix and the 320 tests originally conducted for each isolate. If strains are isolated from diverse ecological habitats such that all six combinations of supermatrix and submatrix become necessary for complete identification, a maximum of 52 tests need to be performed for each strain; the practical difference between the 52 tests of the supermatrix-submatrix scheme and the 61 tests of the inclusive scheme represents at least a 9 . test economy.

\section{LITERATURE CITED}

1. Bascomb, S., S. P. Lapage, M. A. Curtis, and W. R. Willcox. 1973. Identification of bacteria by computer: identification of reference strains. J. Gen. Microbiol. 77:291-315.

2. Buchanan, R. E., and N. E. Gibbons (ed.). 1974. Bergey's manual of determinative bacteriology, 8th ed. The Williams \& Wilkins Co., Baltimore.

3. Dybowski, W., and D. A. Franklin. 1968. Conditional probability and the identification of bacteria: a pilot study. J. Gen. Microbiol, 54:215-229.

4. Friedman, R. B., D. Bruce, J. MacLowry, and V. Brenner. 1973. Computer assisted identification of bacteria. Am. J. Clin. Pathol. 60:395-403.

5. Friedman, R. B., and J. MacLowry. 1973. Computer identification of bacteria on the basis of their antibiotic susceptibility patterns. Appl. Microbiol. 26:314-317.

6. Gyllenberg, H. G. 1965. A model for computer identification of microorganisms. J. Gen. Microbiol. 39:401-405.

7. Hauxhurst, J. D., M. I. Krichevsky, and R. M. Atlas. 1980. Numerical taxonomy of bacteria from the Gulf of Alaska. J. Gen. Microbiol. 120:131-148.
8. Johnson, R. 1979. Computer-aided identification. FDA (Food Drug Adm.) By-Lines 9:235-250.

9. Kaneko, T., R. M. Atlas, and M. I. Krichevsky. 1977. Diversity of bacterial populations in the Beaufort Sea Nature (London) 270:596-599.

10. Kaneko, T., J. Hauxhurst, M. I. Krichevsky, and R. M Atlas. 1978. Numerical taxonomic studies of bacteria isolated from arctic and subarctic marine environments. p. 26-30. In M. W. Loutit and J. A. R. Miles (ed.), Microbial ecology. Springer-Verlag, Berlin.

11. Kaneko, T., M. I. Krichevsky, and R. M. Atlas. 1979. Numerical taxonomy of bacteria from the Beaufort Sea. J. Gen. Microbiol. 110:111-125.

12. Lapage, S. P., S. Bascomb, W. R. Willcox, and M. A. Curtis. 1970. Computer identification of bacteria. Soc. Appl. Bacteriol. Tech. Ser. 4:1-22.

13. Lapage, S. P., S. Bascomb, W. R. Willcox, and M. A. Curtis. 1973. Identification of bacteria by computer: general aspects and perspectives. J. Gen. Microbiol. 77:273290.

14. Sneath, P. H. A., and R. R. Sokal. 1973. Numerical taxonomy. W. H. Freeman \& Co., San Francisco.

14a.Walczak, C. A., and M. I. Krichevsky. 1982. Computeraided selection of efficient identification features and calculation of group descriptors as exemplified by data on Capnocytophaga species. Curr. Microbiol. 7:199-204.

15. Wayne, L. G., E. J. Krichevsky, L. L. Love, R. Johnson, and M. I. Krichevsky. 1980. Taxonomic probability matrix for use with slowly growing mycobacteria. Int. J. Syst. Bacteriol. 30:528-538.

16. Willcox, W. R., S. P. Lapage, S. Bascomb, and M. A. Curtis. 1973. Identification of bacteria by computer: theory and programming. J. Gen. Microbiol. 77:317-330.

17. Willcox, W. R., S. P. Lapage, and B. Holmes. 1980. A review of numerical methods in bacterial identification. Antonie van Leeuwenhoek J. Microbiol. Serol. 46:233299. 